\title{
Efetividade da gamificação em simulador de anestesia odontológica
}

\author{
Matheus A. O. Ribeiro ${ }^{1}$, Cléber G. Corrêa ${ }^{1}$, Fátima L. S. Nunes ${ }^{1}$ \\ ${ }^{1}$ Laboratório de Aplicações de Informática em Saúde - LApIS \\ Escola de Artes, Ciências e Humanidades da Universidade de São Paulo - EACH/USP \\ São Paulo - SP - Brazil \\ \{matheus.alberto.ribeiro, fatima.nunes, cleber.gimenez\} @usp.br
}

\begin{abstract}
Due to the high failure rate in the procedure of applying dental anesthesia, this work presents the gamification process of a simulator of this procedure and its validation. From the literature review and simulator analysis, game elements were listed and implemented. The serious game aims to promote a learning strategy that is more attractive, encouraging and allows training evaluation. Partial results of an experiment performed by volunteers indicate that the gamification process was successful and that the game can be submitted for validation with Dentistry professionals.
\end{abstract}

Resumo. Devido à alta taxa de insucesso no procedimento de aplicação de anestesia odontológica, o presente trabalho apresenta o processo de gamificação de um simulador deste procedimento e sua validação. A partir da revisão literária e análise do simulador, elementos de jogos foram elencados e implementados. O jogo sério objetiva promover uma estratégia de aprendizagem que seja mais atraente, incentivadora e permita a avaliação do treinamento. Resultados parciais de um experimento com voluntários indicam que o processo de gamificação foi bem sucedido e que o jogo pode ser submetido para validação com profissionais da área de Odontologia.

\section{Introdução}

A aplicação de anestesia odontológica é um procedimento com alta taxa de insucesso, inclusive durante o treinamento [Poyade et al. 2014]. Simuladores em Realidade Virtual (RV) desse tipo de procedimento contribuem para a aquisição de conhecimento e habilidades motoras necessárias para a formação de profissionais da área da saúde. Entretanto, muitas vezes são pouco atraentes para os aprendizes. Para tornar tais simuladores virtuais mais atrativos, o processo de gamificação (do inglês gamification) pode ser empregado.

O presente trabalho apresenta como contribuição o estudo, a definição e a validação do processo de gamificação em um simulador com interface háptica para o treinamento do procedimento de aplicação de anestesia odontológica [Ribeiro et al. 2017]. A partir de um experimento, conduzido com voluntários, a efetividade da gamificação foi avaliada considerando o engajamento e a percepção dos jogadores.

\section{Conceitos e trabalhos correlatos}

O termo "jogos sérios" (do inglês serious games) refere-se a uma classe de jogos voltada a simular situações do cotidiano [Michael and Chen 2005]. O processo de incorporação de elementos de jogos em aplicações computacionais com o objetivo de despertar 
o engajamento de um público-alvo é chamado de gamificação (do inglês gamification) [Huotari and Hamari 2012].

Alguns simuladores para treinamento odontológico podem ser encontrados na literatura [Poyade et al. 2014, Ullah and Park 2012]. Apesar do uso de dispositivos hápticos, nenhum deles aplica a gamificação. Em [Vannaprathip et al. 2016], elementos de jogos são utilizados, mas não há o uso de dispositivos hápticos, diminuindo a imersão e o realismo.

Na literatura são encontrados jogos sérios com diversas finalidades, que podem ser classificadas em treinamento de procedimentos, conscientização e reabilitação [Machado et al. 2011]. Na categoria de treinamento de procedimentos se enquadram os jogos que objetivam aperfeiçoar alguma habilidade. O jogo apresentado em [Brazil et al. 2016], por exemplo, promove o treinamento do procedimento de anestesia epidural. Um exemplo de jogo sério para conscientização é apresentado em [Molnar and Kostkova 2014], sendo voltado à conscientização de crianças sobre uso de antibióticos e higiene. Em [Funabashi et al. 2017] é apresentado um jogo sério para reabilitação de pacientes vítimas de acidente vascular encefálico.

Nos jogos sérios encontrados na literatura é possível observar a recorrência de elementos como pontuações e níveis de dificuldade, utilizados para avaliar desempenho e oferecer desafios ao jogador. Para o treinamento de procedimentos na área da saúde, em que exige-se precisão no controle de instrumentos médicos, o uso de dispositivos hápticos é comum. Além dos citados, também são encontrados elementos de jogos como a presença de um alvo destacado, conquistas/troféus e rankings. Tais elementos da gamificação podem ser incorporados em simuladores, com o objetivo de torná-los mais atrativos para os aprendizes. Uma análise mais completa da literatura é apresentada em [Ribeiro et al. 2017].

\section{Gamificação do simulador de anestesia odontológica}

O objetivo do simulador para aplicação de anestesia odontológica é disponibilizar um sistema de RV para treinamento deste procedimento, com uso de dispositivo háptico (Figura 1). Nele, o usuário tem controle de uma seringa e deve direcioná-la até uma região próxima ao nervo alveolar inferior direito, que é o local correto de inserção da anestesia [Corrêa et al. 2017]. Uma esfera virtual foi posicionada nesse local, de modo a destacá-lo. Um modelo de paciente, bem como um cenário simulando um consultório e uma seringa, foram implementados. A cabeça do paciente é composta por diversas camadas (e.g. pele, gengiva, ossos, dentes, etc.) com propriedades de massa e rigidez específicas, utilizadas no cálculo do retorno de força do dispositivo háptico ou resistência que o dentista deve sentir de modo realista [Ribeiro et al. 2017, Corrêa et al. 2017].

A partir da revisão da literatura (Seção 2) e da análise do simulador, elementos de jogos como tempo, pontuações, registro de erros, níveis, ícones e troféus foram implementados [Ribeiro et al. 2017]. A Figura 2 descreve os passos a serem seguidos durante uma jogada (i.e., execução completa do procedimento). Um exemplo pode ser verificado na Figura 3. Inicialmente, o jogador possui liberdade de movimentação da seringa. Após se aproximar da cabeça do paciente (área crítica), a jogada é iniciada. O jogador deve então direcionar a seringa até o local correto de inserção, representado pela esfera verde, com atenção para não perfurar o paciente em local incorreto, o que resulta em punições 


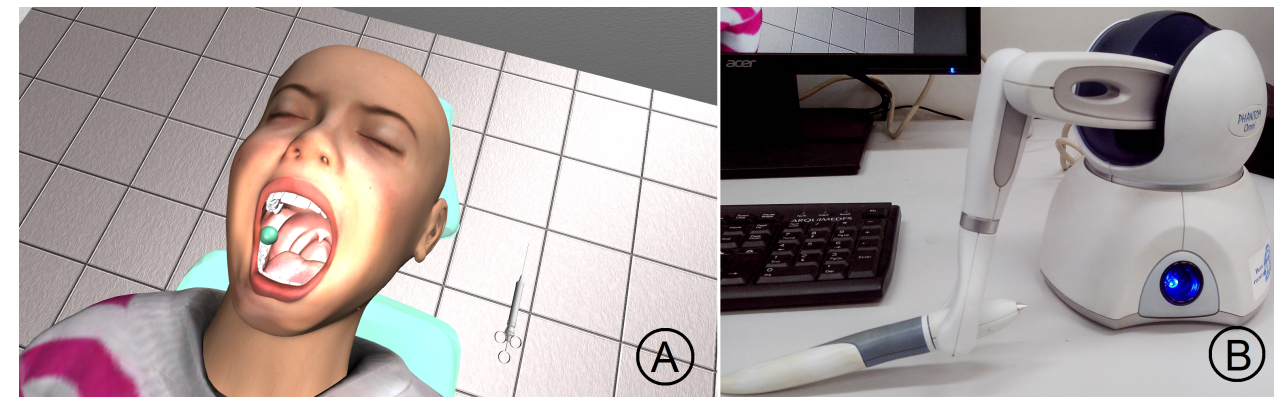

Figura 1. (A) Simulador de aplicação de anestesia no nervo alveolar inferior direito. O local de inserção da agulha é representado pela esfera verde. (B) Dispositivo háptico utilizado para controlar a seringa.

(pontuação de erro é acrescida e causa é registrada). Após acertar o local, o jogador deve penetrar a gengiva com angulação da seringa próxima à ideal (definida pelo especialista) e realizar a liberação do anestésico em profundidade dentro do intervalo permitido. Uma seringa verde indica visualmente ao jogador tais parâmetros, enquanto ícones guiam o jogador visualmente durante as etapas do procedimento. Finalmente, após inserir toda a anestesia, o jogador deve retirar a agulha e afastar a seringa da cabeça do paciente, atingindo o fim da jogada. Após o término, uma tela com os resultados é exibida.

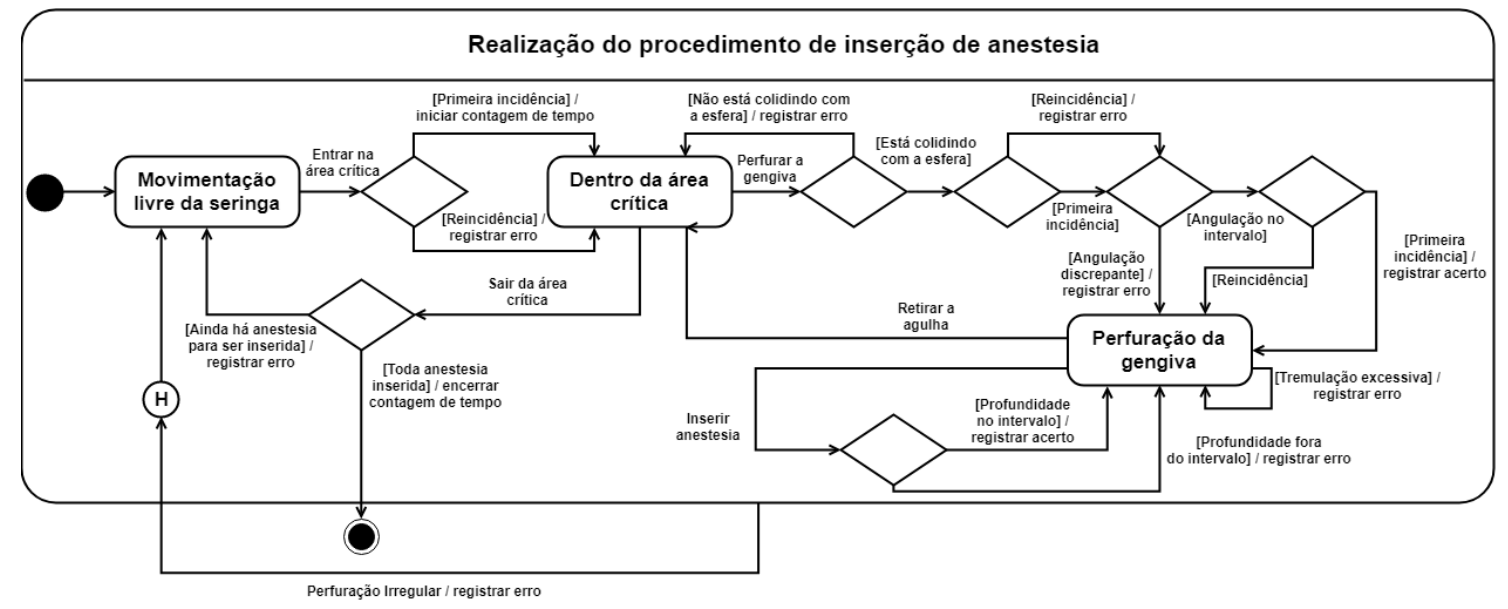

Figura 2. Máquina de estados da Unified Modeling Language (UML) representando todas as etapas do procedimento de aplicação da anestesia.

A fim de tornar a avaliação do desempenho mais detalhada, optou-se pela separação das pontuações entre acertos e erros. Cada erro definido no jogo possui um peso proporcional a sua gravidade em um procedimento real. Acertos são dados se os intervalos pertinentes à angulação da seringa durante a penetração e profundidade de liberação do anestésico forem respeitados. Seus pesos são proporcionais à precisão dessas etapas (i.e., quanto mais próxima da ideal, maior). Tais intervalos, bem como o tamanho do local correto de inserção, podem ser modificados a fim de criar níveis de dificuldade.

Todas as jogadas são incluídas em um histórico, permitindo a avaliação do progresso do jogador. Com o intuito de estimular a competição e oferecer incentivo à aquisição de habilidades, sistemas de ranking e troféus foram adicionados. Para cada nível, restrições de pontuações e tempo podem ser definidas. $\mathrm{O}$ alcance dos objetivos resulta na 


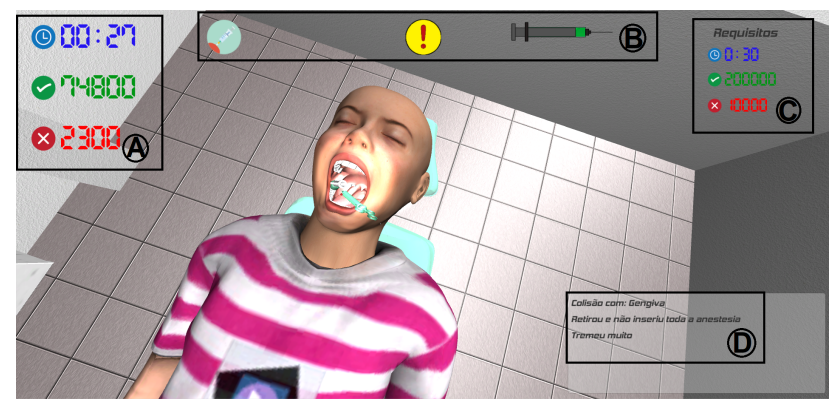

Figura 3. Jogada em andamento. (A) tempo e pontuações de acerto e erro da jogada; (B) ícones de penetração da gengiva, proximidade da cabeça do paciente e quantidade de anestésico restante; (C) requisitos para obtenção de troféus; (D) registro de erros.

obtenção de troféus.

\section{Experimento}

Com o objetivo de verificar a efetividade do processo de gamificação do simulador, um experimento foi realizado com 12 voluntários, que utilizaram tanto a versão gamificada (aqui denominada "jogo") quanto a não gamificada (aqui denominada "simulador"). Para tal, a seguinte hipótese foi estabelecida: "o jogo é mais atrativo aos voluntários e contribui mais para o aprendizado em comparação com o simulador". Considerou-se que a versão gamificada acarretaria em maior engajamento do voluntário e, consequentemente, o sistema seria usado por mais tempo, evidenciando sua efetividade.

O experimento continha três iterações e foi composto pela utilização dos sistemas e preenchimento de questionários de autoeficácia (Tabela 1) com escala Likert de seis níveis [Bandura 2006]. Na primeira iteração, apenas o questionário era respondido. Nas demais, o voluntário assistia a um vídeo de 30 segundos do jogo ou simulador, que descrevia o que devia ser feito (Seção 3). Em seguida, o voluntário dispunha de dez minutos máximos para a utilização do respectivo sistema, podendo parar a qualquer momento. $\mathrm{O}$ tempo de uso de cada sistema foi medido, bem como a quantidade de jogadas realizadas. Ao final da iteração, o questionário era respondido novamente. A fim de evitar possíveis vieses, a ordem de apresentação dos sistemas foi alternada entre os participantes, de modo que metade utilizou o jogo após o simulador, enquanto a outra o utilizou primeiro.

Tabela 1. Perguntas do questionário de autoeficácia.

\begin{tabular}{|c|c|}
\hline $\mathbf{N}^{\mathbf{0}}$ & Pergunta \\
\hline 1 & Sou capaz de utilizar o dispositivo háptico sem dificuldades \\
\hline 2 & Eu conheço o procedimento de aplicação de anestesia odontológica \\
\hline 3 & Sou capaz de realizar a aplicação de anestesia odontológica em pacientes virtuais (usando simuladores computacionais) \\
\hline 4 & Sou capaz de realizar oa aplicação de anestesia odontológica em pacientes reais \\
\hline 5 & Sou capaz de acertar o ponto correto de inserção da agulha para a aplicação de anestesia odontológica (representado pela esfera verde) \\
\hline 6 & Um jogo com elementos de competição (pontuações, rankings, troféus, etc.) me motiva a aperfeiçoar minhas habilidades \\
\hline
\end{tabular}

\section{Resultados e discussão}

A Figura 4A apresenta o tempo que cada voluntário usou cada um dos sistemas. Na média, o jogo foi utilizado por 3 minutos e 11 segundos a mais que o simulador. Considerando 
que o procedimento oferecido pelos dois sistemas, assim como a forma de interação, se mantém nas duas aplicações, o maior tempo de permanência no jogo pode ser indício de maior engajamento. Os voluntários 1 e 2, que apresentaram uma maior discrepância, realizaram diversas jogadas. Já os voluntários 4, 11 e 12 obtiveram uma discrepância menor, devido a terem jogado apenas uma vez, o que pode mostrar menor engajamento.
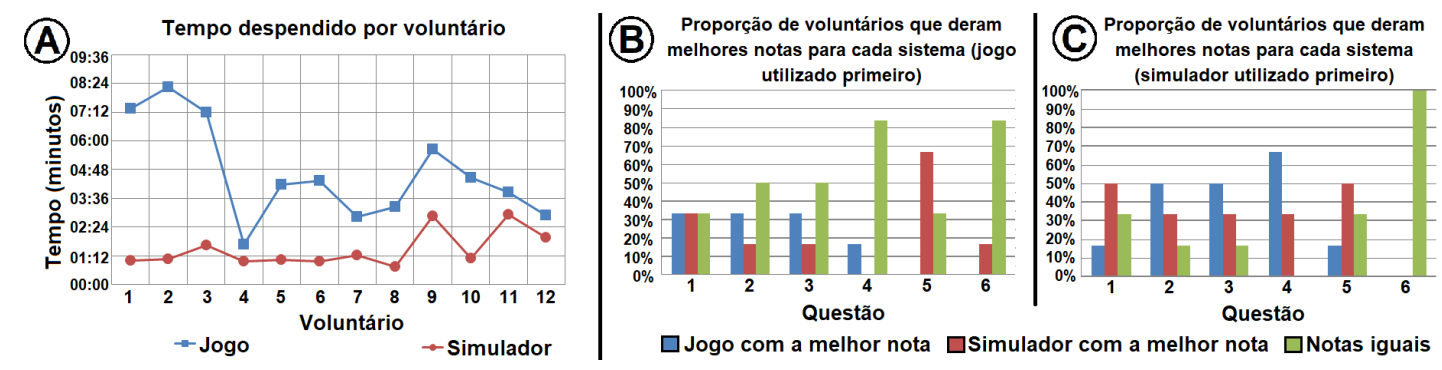

Figura 4. (A) Tempo despendido por cada voluntário para o jogo e o simulador.
Proporção de voluntários que deram melhores notas para cada sistema
para o grupo que utilizou primeiro o jogo (B) e primeiro o simulador (C).

Para o questionário de autoeficácia foram analisados o número de voluntários que deram melhores notas para o jogo ou para o simulador. As Figuras 4B e 4C exibem, respectivamente, as proporções de voluntários que deram melhores notas para cada sistema. Sobre a capacidade de utilização do dispositivo háptico (questão 1) e capacidade de acertar o ponto correto de inserção da agulha (questão 5), a proporção de voluntários que avaliou melhor o simulador foi maior ou igual ao jogo. Isso pode ser explicado pelo fato de o jogo indicar os erros do jogador, o que pode influenciar na percepção do voluntário sobre seu desempenho. Este elemento pode ter contribuído para os valores obtidos em tais questões, levando a uma autoavaliação mais crítica dos voluntários.

Os erros e ícones indicadores no jogo também podem auxiliar no aprendizado (questão 2). Em ambos os grupos, o jogo obteve notas superiores ao simulador. Metade dos voluntários que usaram primeiro o jogo atribuíram notas iguais aos dois sistemas avaliados. Como o jogo foi apresentado primeiro, este fato pode indicar que o conhecimento sobre o procedimento adquirido persistiu, mantendo assim a mesma nota de autoeficácia. Ocorrências similares podem ser observadas para as questões 3 e 4, que abordam a capacidade de realização do procedimento em pacientes virtuais e reais, respectivamente. $\mathrm{O}$ jogo recebeu notas melhores do que o simulador, com grande proporção de empate para quem usou o jogo primeiro. A presença de elementos de avaliação e competição no jogo (questão 6) pode ter deixado os voluntários mais seguros de suas capacitações e motivados a melhorar, o que justifica a melhor avaliação do jogo nessas questões.

\section{Conclusões}

O presente trabalho teve como objetivo apresentar o processo e a avaliação experimental da efetividade da gamificação de um simulador do procedimento de anestesia odontológica, visando tornar o treinamento do mesmo mais atrativo e seguro para os aprendizes. Um experimento, conduzido com voluntários, mostrou que o tempo de utilização do jogo foi maior do que o simulador, fornecendo indícios de maior engajamento proporcionado pelo jogo. A análise do questionário de autoeficácia sugere que a presença dos elementos de jogos auxilia no aprendizado e aumenta a confiança dos usuários. Trabalhos futuros 
incluem a execução de novos experimentos com profissionais da área de Odontologia, que constitui a limitação do presente trabalho. Assim, pode ser possível obter novas evidências da efetividade da gamificação e identificar pontos de melhoria.

\section{Referências}

Bandura, A. (2006). Guide for constructing self-efficacy scales. Self-efficacy beliefs of adolescents, 5:307-337.

Brazil, A. L., Conci, A., Clua, E., Rodriguez-Hernandez, N., Bittencourt, L. K., and Ramos, R. R. (2016). Force modeling and gamification for epidural anesthesia training. In 2016 IEEE International Conference on Serious Games and Applications for Health (SeGAH), pages 1-8.

Corrêa, C. G., Machado, M. A. A. M., Ranzini, E., Tori, R., and Nunes, F. L. S. (2017). Virtual reality simulator for dental anesthesia training in the inferior alveolar nerve block. Journal of Applied Oral Science, 25(4):357-366.

Funabashi, A. M. M., Aranha, R. V., Silva, T. D., Monteiro, C. B. M., Silva, W. S., and Nunes, F. L. S. (2017). AGaR: A VR serious game to support the recovery of poststroke patients. In 2017 19th Symposium on Virtual and Augmented Reality (SVR), pages 279-288.

Huotari, K. and Hamari, J. (2012). Defining gamification: A service marketing perspective. In Proceeding of the 16th International Academic MindTrek Conference, pages 17-22, New York, NY, USA. ACM.

Machado, L. S., Moraes, R. M., Nunes, F. L. S., and Costa, R. M. E. M. (2011). Serious games baseados em realidade virtual para educação. Revista Brasileira de Educação Médica, 35:254 - 262.

Michael, D. R. and Chen, S. L. (2005). Serious Games: Games That Educate, Train, and Inform. Muska \& Lipman/Premier-Trade.

Molnar, A. and Kostkova, P. (2014). Gaming to master the game - game usability and game mechanics. In 2014 IEEE 3rd International Conference on Serious Games and Applications for Health (SeGAH), pages 1-7.

Poyade, M., Lysakowski, A., and Anderson, P. (2014). Development of a haptic training simulation for the administration of dental anaesthesia based upon accurate anatomical data. In: EuroVR 2014 - Conference and Exhibition of the European Association of Virtual and Augmented Reality: The Eurographics Association.

Ribeiro, M. A. O., Corrêa, C. G., and Nunes, F. L. S. (2017). Gamification as a learning strategy in a simulation of dental anesthesia. In 2017 19th Symposium on Virtual and Augmented Reality (SVR), pages 271-278.

Ullah, F. and Park, K. (2012). Visual, haptic, and auditory realities based dental training simulator. In 2012 International Conference on Information Science and Applications, pages $1-6$.

Vannaprathip, N., Haddawy, P., Suebnukarn, S., Sangsartra, P., Sasikhant, N., and Sangutai, S. (2016). Desitra: A simulator for teaching situated decision making in dental surgery. In Proceedings of the 21st International Conference on Intelligent User Interfaces (IUI), pages 397-401, New York, NY, USA. ACM. 